\title{
O registro em prontuário coletivo no trabalho do psicólogo na Estratégia Saúde da Família
}

Patrícia Matte Rodrigues. Secretaria de Município da Saúde de Lages.

Patrícia Paraboni. Universidade Federal de Santa Maria.

Dorian Mônica Arpini. Universidade Federal de Santa Maria.

Catheline Rubim Brandolt. Universidade Federal de Santa Maria.

Jessica Vaz Lima. Universidade Federal de Santa Maria.

Pâmela Kurtz Cezar. Prefeitura Municipal de Santa Maria.

\section{Resumo}

Este artigo constitui um relato de experiência, o qual aborda e problematiza o registro de informações nos prontuários coletivos em equipes de Saúde da Família pelos profissionais de Psicologia vinculados a um Programa de Residência Multiprofissional. O Conselho Federal de Psicologia destaca que o psicólogo, em serviço multiprofissional, deve fazer uso do prontuário único, registrando apenas as informações necessárias aos objetivos do trabalho. Essa questão é complexa uma vez que envolve aspectos éticos e de sigilo profissional. Entretanto, entende-se que tal temática precisa ser discutida pelos profissionais de Psicologia e no cotidiano das equipes, para qualificar o cuidado em saúde.

Palavras-chave: psicologia; saúde da família; sistema único de saúde; registros de saúde pessoal; troca de informação em saúde.

\begin{abstract}
The record on collective electronic chart in the context of the psychologist's work in Family Health Strategy. This study is an experience report that approaches and problematizes the act of recording information on collective charts in the context of Family Health teams carried out by Psychology professionals of a Multiprofessional Residency Program. The Federal Council of Psychology highlights the psychologist that works in a public service should register the information on the chart that the other professionals have access to, recording only information related to the aim of his task. This is a complex subject, once it involves ethical and professional secrecy aspects. Such aspects must be discussed by Psychology professionals in the context of health teams, so as to improve health care.
\end{abstract}

Keywords: psychology; family health; unified health system; personal health records; health information exchange.

\section{Resumen}

El registro en la historia clínica colectiva en el trabajo del psicólogo en la Estrategia de Salud de la Familia. Este artículo se constituye en un relato de experiencia, el cual aborda y problematiza el registro de informaciones en las historias clínicas colectivas, en equipos de Salud de la Familia, por los profesionales de Psicología vinculados a un Programa de Residencia Multiprofesional. El Consejo Federal de Psicología destaca que el psicólogo en servicio multiprofesional, debe hacer uso de una historia clínica única, registrando solamente las informaciones necesarias para los objetivos del trabajo. Esta cuestión es compleja, una vez que implica aspectos éticos y de secreto profesional. Sin embargo, se entiende que esta temática debe ser discutida por los profesionales de la Psicología y en el cotidiano de los equipos, para calificar el cuidado de la salud.

Palabras clave: psicología; salud de la familia; sistema único de salud; registros personales de salud; intercambio de información de salud. 


\section{Prontuário em equipe multiprofissional: informações necessárias e qualidade dos registros}

No Brasil, a proposta de reorganização da Atenção Primária à Saúde (APS) tem na Saúde da Família sua principal estratégia para a mudança do modelo de saúde. Nesse contexto, uma das ferramentas presentes no trabalho das equipes de Estratégia Saúde da Família (ESF) e que tem se colocado como um dos grandes desafios aos profissionais são os prontuários multiprofissionais, os quais devem conter informações importantes acerca da situação de saúde dos usuários/ famílias daquele serviço.

Conforme Pinto (2006), inicialmente a redação dos registros nos prontuários era realizada unicamente pelo médico, entretanto, com o avanço das áreas paramédicas (da qual faz parte a Psicologia) e a exigência interdisciplinar, o prontuário passa a ser redigido de forma coletiva. Os registros devem ser constantemente atualizados pelos profissionais da equipe, uma vez que esta é, por vezes, bastante rotativa.

A Política Nacional da Atenção Básica (PNAB) define que os profissionais que atuam na ESF devem garantir a qualidade do registro das atividades nos sistemas nacionais de informação da Atenção Básica (Portaria $n^{\circ} 2.488$, de 21 de outubro de 2011). Demarzo, Oliveira e Gonçalves (s/d) apontam que o registro da prática clínica na Atenção Primária à Saúde apresenta finalidades como a longitudinalidade e a coordenação do cuidado, os quais diferem de formatos geralmente empregados na atenção especializada e hospitalar. A prática na APS pode ser compreendida dentro de um conjunto articulado e integrado de ações com o intuito de promover a atenção integral à saúde aos usuários (Demarzo et al., s/d).

Segundo Santos e Ferreira (2012) a Saúde da Família possui, na utilização do prontuário, um dispositivo estratégico para a realização do cuidado, na medida em que pode garantir a manutenção do registro das informações e o acesso às ações realizadas pela equipe multiprofissional (Malaman, Costa, Monteiro, \& Figueira, 2012; Pereira et al., 2008). Assim, entende-se que os prontuários utilizados pelas equipes de ESF contemplam diversas informações sobre os usuários e suas famílias, incluindo consultas, exames realizados, medicamentos utilizados, situação de saúde, encaminhamentos, entre outras informações, constituindo um guia sobre a história clínica do usuário.
O prontuário da família representa uma rica fonte de informações para a equipe de saúde, no sentido de qualificar o atendimento à população e desenvolver a coordenação da atenção. A capacidade de integração das informações de cunho individual, familiar e/ ou social possibilita a qualificação das ações de saúde (Santos \& Ferreira, 2012). Também se constitui num instrumento de respaldo ético e legal aos profissionais responsáveis pelo cuidado (Malaman et al., 2012; Pereira et al., 2008) e elemento fundamental em casos de auditoria (Pereira et al., 2008). Portanto, conforme Malaman e colaboradores (2012), em caso de registro deficitário e inadequado a assistência prestada ao usuário pode ficar comprometida.

A segurança da informação é bastante delicada, mas no caso do prontuário da família, ainda pouco discutida. Um modelo ideal de prontuário da família deve considerar a segurança das informações e as questões éticas que abrangem a área da informação em saúde, seja em relação ao acesso dos profissionais, como dos próprios membros da família, visto que teriam acesso às informações de outros familiares (Santos \& Ferreira, 2012).

No entender de Santos e Ferreira (2012) frente à necessidade de integração dos dados e disponibilidade das informações, o uso de tecnologias de informação e comunicação pode se constituir como um elemento estratégico na Saúde da Família. Pinto (2006) esclarece que o Prontuário Eletrônico do Paciente (PEP) é um "documento de referência no processo de comunicação intra e entre a equipe de saúde e o paciente" (p. 34). O PEP apresenta estrutura física (dados relativos ao paciente, convênio e internação, assim como dados referentes à saída do paciente das organizações de saúde) e lógica (descrição das informações sobre o estado de saúde do paciente, além de relatórios e evoluções). É necessário que o PEP seja bem redigido no sentido da qualidade das informações, caso contrário poderá provocar transtornos no fluxo de informações e de comunicação, causando danos ao paciente e equipe de saúde.

Em relação ao registro em prontuário coletivo no trabalho do psicólogo, verificou-se escassez de estudos que relatassem e/ou avaliassem essa prática no contexto da APS. Deste modo, no que segue serão apresentadas algumas recomendações do Conselho Federal de Psicologia (CFP), referentes às orientações sobre o registro em prontuários multiprofissionais. A partir disso, pretende-se abordar e problematizar o 
registro de informações nos prontuários coletivos em equipes de Saúde da Família a partir das vivências de profissionais de Psicologia vinculados a um Programa de Residência Multiprofissional.

\section{O registro do trabalho do psicólogo em prontuário coletivo}

Este artigo resulta de reflexões realizadas por psicólogas vinculadas a um Programa de Residência Multiprofissional (PRM) de uma instituição pública de ensino superior, de uma cidade do interior do estado do Rio Grande do Sul. Desde o ano de 2009, com a primeira turma do PRM, o núcleo de psicologia tem problematizado suas práticas no campo da Atenção Primária à Saúde.

Atualmente, as psicólogas residentes atuam em duas Unidades de ESF, as quais contam com a equipe mínima de saúde composta por médico, enfermeira, técnico de enfermagem, agentes comunitários de saúde e equipe de saúde bucal, com dentista e auxiliar de consultório dentário (Portaria $\mathrm{n}^{\circ} 2.488$, de 21 de outubro de 2011). Além desses profissionais, essas equipes contam com a presença de residentes de diferentes núcleos como educação física, enfermagem, fisioterapia, nutrição, odontologia, psicologia e serviço social. Destaca-se que as psicólogas vinculadas ao PRM participam também de Tutoria e Preceptoria de Núcleo, espaços semanais e/ou quinzenais de supervisão/orientação acadêmica, nos quais são problematizadas as práticas cotidianas de atuação da psicologia no contexto da APS.

Por conseguinte, na prática das psicólogas residentes, o registro no prontuário coletivo é realizado de forma concisa, adequando informações necessárias para o entendimento do que foi abordado pelo usuário durante seu atendimento, de modo que os demais profissionais da equipe consigam compreender os aspectos psicossociais envolvidos. No entanto, questões importantes que são trazidas ao psicólogo, como situações de violência doméstica, violência sexual, questões jurídicas, entre outras, são aspectos que exigem do profissional da psicologia uma reflexão mais atenta sobre a prática do registro de informações.

Diante destas questões, é fundamental o diálogo com a equipe para que se possa definir os pontos principais a serem descritos no prontuário e assim estabelecer uma linguagem acessível com as informações mais importantes para a coordenação e continuidade da atenção aos usuários. Não raro, nos atendimentos prestados aos usuários, é necessário elucidar aos mesmos a necessidade de compartilhar as suas informações pessoais com outros profissionais da equipe de saúde em determinadas situações. Ressalta-se que através dos encontros de Tutoria e Preceptoria de Núcleo foi possível estabelecer métodos para definição de condutas profissionais, como a do registro de informações pessoais em prontuários coletivos. Cabe salientar, que houve casos em que foi necessário o rompimento do sigilo profissional, realizado após os cuidados descritos acima.

Por conseguinte, alguns questionamentos se fizeram presentes no cotidiano de atuação das psicólogas residentes, quais sejam: como compartilhar aspectos que foram revelados de forma sigilosa, mas que são fundamentais para a abordagem da equipe em relação ao usuário e sua família? De que modo o psicólogo deve compartilhar as informações sobre os usuários sem ferir seu Código de Ética Profissional? Como superar a compreensão de que o usuário está vinculado ao serviço e não unicamente ao profissional, como no caso do exercício profissional em consultório particular? Além do prontuário coletivo, o psicólogo deveria manter um registro individual dos casos?

O que recomenda o Conselho Federal de Psicologia (CFP) sobre o registro do trabalho do psicólogo em prontuário coletivo? O CFP, através da Resolução n 001/2009, em seu Art. $1^{\circ}$, torna obrigatório o registro documental sobre a prestação de serviços psicológicos, os quais não puderem ser mantidos prioritariamente sob a forma de prontuário psicológico. Além disso, destaca que o registro documental, em papel ou informatizado, tem caráter sigiloso e se constitui como um conjunto de informações que visa contemplar de forma sucinta o trabalho prestado, a descrição e a evolução da atividade, assim como os procedimentos técnico-científicos adotados.

A Resolução 001/2009 ainda prevê que no caso do registro documental ser realizado na forma de prontuário é necessário registrar as informações recomendadas acima e, além disso, fica garantido ao usuário ou representante legal o acesso integral às informações registradas pelo psicólogo em seu prontuário. Em seu Art. $6^{\circ}$ o documento prevê que quando em serviço multiprofissional, o registro do psicólogo deve ser realizado em prontuário único. $\mathrm{E}$, ainda esclarece que: "Devem ser registradas apenas as informações necessárias ao cumprimento dos objetivos do trabalho" (Resolução 001/2009). Essa mesma recomendação encontra-se 
no art. $12^{\circ}$ do Código de Ética Profissional do Psicólogo (CFP, 2005), e em seu art. $6^{\circ}$, item b, consta que o psicólogo deverá "compartilhar somente informações relevantes para qualificar o serviço prestado, resguardando o caráter confidencial das comunicações, assinalando a responsabilidade, de quem as receber, de preservar o sigilo" (p. 12). Cabe ressaltar que o sigilo sobre as informações compartilhadas é ainda algo que precisa ser melhor discutido e trabalhado com as equipes, conforme apontado por Santos e Ferreira (2012).

No que concerne à qualidade dos registros, entende-se ser possível buscar orientações na Resolução $n^{\circ}$ 007/2003, na qual foi instituído um Manual de Elaboração de Documentos Escritos produzidos por psicólogos. Este manual está direcionado para a escrita de documentos decorrentes de avaliação psicológica. Embora não seja essa a demanda dos psicólogos no contexto da ESF, esse documento traz reflexões referentes à preocupação com a qualidade do registro das informações em prontuário multiprofissional, portanto recorreu-se a ele para tentar encontrar orientações que auxiliassem na redação de prontuários coletivos. Cabe esclarecer também que o manual em questão visa orientar o profissional na confecção de documentos, assim como fornecer subsídios éticos e técnicos necessários à elaboração qualificada da comunicação escrita. Consta que:

O documento deve, na linguagem escrita, apresentar uma redação bem estruturada e definida, expressando o que se quer comunicar. Deve ter uma ordenação que possibilite a compreensão por quem o lê, o que é fornecido pela estrutura, composição de parágrafos ou frases, além da correção gramatical (Resolução nº 007/2003).

Quanto à linguagem dos documentos, orienta que ela seja "precisa, clara, inteligível e concisa, ou seja, deve-se restringir pontualmente às informações que se fizerem necessárias, recusando qualquer tipo de consideração que não tenha relação com a finalidade do documento específico" (Resolução n 007/2003).

A linguagem que cada especialista utiliza na redação do prontuário, está diretamente relacionada com a sua área de atuação e sua especificidade. Entretanto, conforme sublinha Pinto (2006), já se percebe a necessidade de uma linguagem comum, uma vez que o prontuário visa favorecer a comunicação entre a equipe de saúde, mas também entre a mesma e o paciente. É neste sentido que a autora defende a necessidade do uso de uma linguagem mais próxima do usuário. Essas observações devem orientar o registro de informações no formato eletrônico ou em papel. Entretanto, no caso dos profissionais da psicologia, o CFP, através da Resolução n 007/2003, orienta que "o emprego de frases e termos deve ser compatível com as expressões da linguagem profissional, garantindo a precisão da comunicação, evitando a diversidade de significações da linguagem popular, considerando a quem o documento será destinado" (Resolução nº 007/2003).

Quanto aos registros nos prontuários coletivos, entende-se que não se trata de utilizar uma linguagem do senso comum/popular, mas que as informações técnicas contidas no prontuário não devem ficar muito distantes do entendimento da equipe e usuários, afinal, conforme orienta o próprio CFP, é preciso considerar a quem o documento está sendo destinado. Ou seja, a linguagem técnica deveria estar acompanhada de uma explicação compatível, que permita o entendimento do que está sendo colocado para os demais integrantes da equipe e o mais próximo possível para os usuários. Se o profissional apenas fizer uso de códigos, signos e expressões próprias do núcleo da psicologia sem uma explicação adequada e acessível poderá dificultar o acesso à compreensão das informações registradas, prejudicando a qualidade e coordenação da atenção e cuidado aos usuários. Assim, ao utilizar uma linguagem mais clara, o psicólogo estará prezando por uma comunicação de maior qualidade tanto com a equipe, quanto com o usuário, indo ao encontro do que é proposto pelos diversos autores anteriormente citados.

Nesse sentido, é preciso levar em consideração o que Mesquita e Deslandes (2010) propõem, ou seja, que o prontuário deve ser uma construção coletiva e deve estar a serviço da comunicação. Para além de um instrumento jurídico ele deve constituir um mediador da comunicação intraequipe e da equipe com o usuário. O sigilo e a linguagem técnica não devem ser uma justificativa para reforçar o poder do conhecimento, do domínio sobre a informação e status profissional. Assim, entende-se que o registro de informações em prontuários tem sido um grande desafio ao trabalho dos psicólogos nos diferentes contextos de saúde. Cabe ressaltar, que esse desafio tem sido potencializado, já que a formação em psicologia não tem discutido, de forma suficiente, o registro em prontuários coletivos, apesar da crescente inserção de psicólogos em equipes de saúde e da assistência social.

Destaca-se ainda que as discussões e orientações realizadas em Tutorias e Preceptorias de Núcleo foram 
importantes para que cada residente da Psicologia fosse encontrando uma forma de fazer seu registro, mas uma questão permaneceu comum, a de que o registro no prontuário coletivo é tarefa importante no trabalho das residentes na APS, além de tal atividade exigir tempo e atenção. Com isso, foi necessário a cada residente organizar sua carga horária, incluindo nela o tempo suficiente para escrita, bem como, local adequado que proporcionasse melhor qualidade no registro nos prontuários.

Portanto, toda atividade dos profissionais de Psicologia envolvendo o usuário tem sido registrada no prontuário coletivo, com informações como: o motivo da procura; se este usuário está vinculado a outro serviço na rede de saúde mental (Centro de Atenção Psicossocial - CAPS, Ambulatório de Saúde Mental) e assistência social (Centro de Referência de Assistência Social - CRAS, Centro de Referência Especializado de Assistência Social - CREAS). Salienta-se também que no caso de o usuário ter sido encaminhado para outro serviço é realizada a tentativa de contato com o mesmo e o contato registrado em prontuário, com objetivo de manter a longitudinalidade, coordenação e continuidade do cuidado. Quando em acompanhamento na ESF registram-se todos os contatos telefônicos realizados para o usuário, seja para agendar a visita domiciliar ou seu atendimento; registram-se também os contatos com familiares, responsáveis e/ou a escola; os encaminhamentos realizados, bem como o término do acompanhamento e o motivo que o teria levado a tal decisão também são registrados.

É importante salientar que essa experiência de resgatar o caminho percorrido pelo usuário na rede de serviços à saúde, evidenciou que muitas vezes não há registro adequado das informações, ou por vezes as informações encontradas nos prontuários são escassas e nem sempre revelam o que foi abordado no atendimento, ou ainda no caso da psicologia, as informações mantem-se em propriedade do profissional, sem registro e indisponível no prontuário coletivo do usuário.

Considerando o exposto, é necessário ressaltar que a inserção da Psicologia no contexto da APS é uma experiência relativamente nova para o psicólogo. Apenas no ano de 2008, com a instituição dos Núcleos de Apoio à Saúde da Família (NASF), que a Psicologia passou a ser reconhecida oficialmente como profissão integrante da Atenção Primária à Saúde (Cezar, Rodrigues, \& Arpini, 2015; Jimenez, 2011). Neste sentido, é importante que ocorra a reflexão de que o usuário, no contexto da APS, não é um paciente exclusivo do psicólogo, mas alguém que tem como referência um serviço e uma equipe. Tal aspecto torna-se um desafio ao psicólogo, mas também aos demais integrantes da equipe de saúde. Durante muito tempo os profissionais tinham o entendimento de que as informações contidas no prontuário eram de sua propriedade ou da instituição, mas atualmente se entende que o prontuário é um documento de comunicação entre os profissionais responsáveis pelo cuidado, o usuário e o serviço de saúde (Secretaria de Estado de Saúde, 2007).

O formato e o conteúdo a ser utilizado nos registros pelas residentes de psicologia, no contexto da ESF, tornaram-se um desafio cotidiano. Houve, inicialmente, o conflito entre a preocupação em descrever de forma detalhada o acompanhamento do usuário e sua família e a responsabilidade profissional de oferecer informações relevantes para o cuidado ao usuário, considerando o trabalho em equipe realizado na ESF.

Durante os encontros de Tutoria e Preceptoria de Núcleo uma das discussões desenvolvidas refletia o desafio que as residentes possuíam em realizar uma atuação psicológica orientada pelo compromisso social, condizente com os princípios do Sistema Único de Saúde (SUS) como é proposto por Lima (2005). Nesse sentido, entende-se que uma atuação mais adequada a nível de APS abrange a discussão dos diferentes elementos que envolvem essa atuação, como é o caso do registro em prontuários multiprofissionais.

No que tange a discussão sobre prontuários é necessário destacar também que atualmente os serviços de saúde, no âmbito do SUS, se encontram em transição para o uso de prontuários eletrônicos. O Sistema de Informação da Atenção Básica (SIAB) foi substituído pelo Sistema de Informação em Saúde para Atenção Básica (SISAB), instituído pela Portaria 1.412 em 10 de julho de 2013. A operacionalização do SISAB é realizada por meio da estratégia denominada e-SUS Atenção Básica (e-SUS AB). Essa transição atende aos diferentes cenários de informatização e conectividade nas unidades de Atenção Primária à Saúde, em âmbito nacional. A estratégia e-SUS AB permite o registro eletrônico de informações em saúde de forma individualizada, possibilitando o acompanhamento do histórico de atendimentos de cada usuário, assim como a produção de cada profissional. Outro diferencial do e-SUS $A B$ é a integração com diversos sistemas de informação oficiais existentes no contexto da APS (Ministério da Saúde, 2014). 
Destaca-se que o município, no qual as psicólogas desenvolveram suas práticas enquanto residentes multiprofissionais utiliza desde o ano de 2014 um Prontuário Privado que migra os dados para o e-SUS AB. Nesse sentido, o processo de transição do registro em papel para o registro eletrônico foi pauta de diversas reuniões de equipe, tendo em vista as dificuldades vivenciadas pelos profissionais, que diziam respeito ao próprio manuseio do sistema informatizado, como o processo de registro das informações.

Deste modo, é possível compreender que o prontuário eletrônico visa facilitar os registros, a leitura e compreensão dos mesmos, bem como a comunicação e a construção das redes, de forma a consolidar o prontuário coletivo. No entanto, muitas questões levantadas nesse artigo ainda permanecem em aberto e necessitam de reflexão, pois os aspectos envolvendo o sigilo e o detalhamento das informações não serão solucionados por via eletrônica, assim como as dificuldades de se trabalhar em equipe e de como as equipes lidam com as informações dos usuários.

Cabe ressaltar também que no prontuário eletrônico disponível para a APS outros desafios se colocam para o núcleo profissional. Considerando que a psicologia, entre outras profissões, não tem integrado a equipe mínima da ESF, há um risco de que os espaços para o registro de atividades do serviço fiquem restritos ao agendamento de consultas. Serão, portanto, necessárias orientações e talvez reformulações no formulário e-SUS para que efetivamente outras atividades desenvolvidas pela psicologia ganhem visibilidade (como por exemplo: acolhimentos, visitas domiciliares, grupos terapêuticos, aconselhamentos em testes rápidos) na APS, pois estas ações ainda são registradas no prontuário eletrônico como prática apenas da atenção secundária.

Neste cenário, ainda há muitas questões críticas a serem enfrentadas e solucionadas no contexto do SUS. Têm-se assim importantes desafios, e as equipes de saúde precisam dedicar tempo para discutir e buscar novos encaminhamentos para os mesmos. É importante destacar ainda que as reuniões de equipe das ESF podem constituir um espaço potente no compartilhamento dos aspectos que envolvem o prontuário coletivo e a relação entre o conhecimento da equipe e os registros da situação referente ao usuário, assim como no que abarca as especificidades do registro de cada núcleo profissional.

\section{Considerações finais}

As problematizações teóricas e as discussões em Tutoria e Preceptoria de Núcleo da Psicologia não extinguem todas as questões frente ao registro das informações em prontuários coletivos. Essa prática requer constantes reflexões por parte de toda a equipe de saúde. As dúvidas ainda são frequentes no cotidiano de atuação e acredita-se que são compartilhadas por outros colegas que atuam em equipes de saúde. Como apontado anteriormente, questões como sigilo e aspectos éticos permeiam essas decisões e certamente preocupam os profissionais que se encontram em atuação na APS.

$\mathrm{Na}$ tentativa de solucionar o impasse entre a questão ética do sigilo e o registro das informações dos usuários/famílias, os profissionais devem efetivar espaços de discussão de caso, com objetivo de analisar cada situação. Da mesma forma, é indispensável que o diálogo ocorra também com o usuário/família, a fim de esclarecer os motivos que levam o profissional a romper o sigilo. O prontuário coletivo é mais que um simples instrumento de registro, é também parte importante da comunicação entre os profissionais da mesma equipe e da rede como um todo. Se há fragilidade na comunicação entre os profissionais, possivelmente haverá deficiências no registro do prontuário e na coordenação do cuidado as famílias.

As tentativas de buscar subsídios para ampliar o conhecimento em relação ao tema, assim como aliviar as angústias em relação ao mesmo, evidenciaram que não existem muitos estudos nessa temática, em especial referente ao núcleo de psicologia. No entanto, é possível destacar que esse tem sido um tema presente entre os profissionais da saúde e que certamente é pauta dos desafios que se encontram inseridos no cotidiano daqueles que integram os serviços do SUS.

Assim, entende-se que os psicólogos não podem mais se restringir aos registros individuais e de propriedade privada, tendo em vista que esse modelo que foi hegemônico não atende mais as necessidades do atual sistema de saúde, no qual muitos psicólogos têm se inserido e muitos Programas de Residência Multiprofissional tem se implementado. Considerando que as Residências em Saúde têm como objetivo qualificar a formação dos profissionais dentro dos pressupostos do SUS, se acredita ser uma tarefa inerente a essa experiência manter a discussão sobre o registro das informações em prontuários coletivos. 
Salienta-se que as vivências aqui relatadas não pretendem definir um modelo padrão de registro em prontuário coletivo para apresentar aos leitores. Contudo, se espera que as reflexões apresentadas e as tentativas de pensar estratégias para melhor lidar com essa exigência possam ser compartilhadas com outros colegas, para que seja possível ampliar o diálogo e encontrar outros caminhos para qualificar o cuidado com o registro das informações no contexto do SUS. Assim, sugere-se a realização de outros estudos envolvendo a temática, bem como se destaca a importância de incluir já na graduação em psicologia a questão dos registros em prontuários coletivos, evitando que os profissionais se sintam despreparados para essa tarefa, quando de sua inserção profissional.

\section{Referências}

Cezar, P. K., Rodrigues, P. M., \& Arpini, D. M. (2015). A Psicologia na Estratégia de Saúde da Família: Vivências da Residência Multiprofissional. Psicologia: Ciência e Profissão, 35(1), 211-224. doi: 10.1590/1982-3703000012014

Conselho Federal de Psicologia. (2005). Código de Ética Profissional do Psicólogo. Brasília, DF: Autor. Recuperado de http://site.cfp.org. br/wp-content/uploads/2012/07/codigo-de-etica-psicologia.pdf

Demarzo, M. M. P., Oliveira, C. A., \& Gonçalves, D. A. (s/d). Prática clínica na Estratégia Saúde da Família - organização e registro. Recuperado de http://www.unasus.unifesp.br/biblioteca_virtual/ esf/2/unidades_conteudos/unidade $15 \mathrm{~m} /$ unidade $15 \mathrm{~m}$.pdf

Jimenez, L. (2011). Psicologia na Atenção Básica à saúde: demanda território e integralidade. Psicologia e Sociedade, 23(spe), 129-139. doi: 10.1590/S0102-71822011000400016

Lima, M. (2005). Atuação psicológica coletiva: uma trajetória profissional em Unidade Básica de Saúde. Psicologia em Estudo, 10(3), 431-440. doi: 10.1590/S141373722005000300011

Malaman, A. O., Costa, A. C., Monteiro, M. S., \& Figueira, S. M. A. (2012). A qualidade dos registros em prontuários em Unidades
Básicas de Saúde de São Paulo. Recuperado de http://www.convibra.com.br/upload/paper/2012/61/2012_61_4265.pdf.

Mesquita, A. O. M., \& Deslandes, S. F. (2010). A construção dos prontuários como expressão da prática dos profissionais de saúde. Saúde e Sociedade, 19(3), 664-673. doi: 10.1590/ S0104-12902010000300017

Ministério da Saúde (2014). Secretaria de Atenção à Saúde. e-SUS Atenção Básica: manual do Sistema com Coleta de Dados Simplificada. Brasília, DF: Ministério da Saúde.

Pereira, A. T. S., Noronha, J., Cordeiro, H., Dain, S., Pereira, T. R., Cunha, F. T. S., \& Costa Jr., H. (2008). O uso do prontuário familiar como indicador de qualidade da atenção nas unidades básicas de saúde. Recuperado de http://www.scielo.br/pdf/csp/v24s1/17.pdf

Pinto, V. B. (2006). Prontuário eletrônico do paciente: documento técnico de informação e comunicação do domínio da saúde. Recuperado de http://www.repositorio.ufc.br/bitstream/riufc/10074/1/2006_art_ vbpinto.pdf

Portaria no 2.488. (2011, 21 de Outubro) Aprova a Política Nacional de Atenção Básica, estabelecendo a revisão de diretrizes e normas para a organização da Atenção Básica, para a Estratégia Saúde da Família (ESF) e o Programa de Agentes Comunitários de Saúde (PACS). Brasília, DF: Ministério da Saúde. Recuperado de http://bvsms.saude.gov.br/bvs/saudelegis/gm/2011/ prt2488_21_10_2011.html.

Resolução no 001 (2009, 30 de março). Dispõe sobre a obrigatoriedade do registro documental decorrente da prestação de serviços psicológicos. Brasília, DF: Conselho Federal de Psicologia.

Resolução n 007 (2003, 14 de junho). Institui o Manual de Elaboração de Documentos Inscritos produzidos pelo psicólogo decorrentes de avaliação psicológica e revoga a Resolução no 17/2002. Brasília, DF: Conselho Federal de Psicologia.

Santos, C., \& Ferreira, J. B. B. (2012). O prontuário da família na perspectiva da coordenação da atenção à saúde. Physis Revista de Saúde Coletiva, 22(3), 1121-1137. doi: 10.1590/ S0103-73312012000300015

Sarti, T. D., Anderson, M. I. P., \& Marto, R. H. (2008). Registros clínicos e atenção primária: reflexões à abordagem biopsicossocial. Revista APS, Juiz de Fora/MG, 11(4), 413-20. Recuperado de http://aps.ufjf. emnuvens.com.br/aps/article/view/275/139

Secretaria de Estado de Saúde (2007). Manual do prontuário de Saúde da Família. Belo Horizonte, MG: SES/MG. 
Patrícia Matte Rodrigues, Mestre em Psicologia pela Universidade Federal de Santa Maria (UFSM), é Psicóloga da Secretaria de Município da Saúde de Lages (SC), Integrante do Núcleo de Apoio à Saúde da Família (NASF).

Endereço para correspondência: Universidade Federal de Santa Maria. Programa de Pós-Graduação em Psicologia - Avenida

Roraima $\mathrm{n}^{\circ} 1000$, prédio 74B, sala 3208. Cidade Universitária. Bairro Camobi. CEP: 97105-900 - Santa Maria (RS). E-mail: patricia_mr@yahoo.com.br

Patrícia Paraboni, Doutora em Teoria Psicanalítica pela Universidade Federal do Rio de Janeiro (UFRJ), Pós-Doutoranda e Professora Colaboradora/Voluntária do Programa de PósGraduação em Psicologia da Universidade Federal de Santa

Maria (UFSM) e Tutora do Núcleo da Psicologia do Programa de Residência Multiprofissional Integrada em Sistema Público de Saúde, ênfase na Atenção Básica da Universidade Federal de Santa Maria (UFSM). Bolsista CAPES. E-mail: pparaboni@yahoo.com.br

Dorian Mônica Arpini, Doutora em Psicologia Social pela Pontifícia Universidade Católica de São Paulo (PUC/SP), Pós-Doutorado em Psicologia pela Universidade de Lisboa (ULisboa), Portugal, é Docente do Departamento de Psicologia e do Programa de

Pós-graduação em Psicologia da Universidade Federal de Santa Maria (UFSM), Tutora de Núcleo do Programa de Residência Multiprofissional Integrada em Sistema Público de Saúde (UFSM).

E-mail: monica.arpini@gmail.com

Catheline Rubim Brandolt, Especialista em Gestão e Atenção de Sistema Público de Saúde (Atenção Básica/ Estratégia Saúde da Família) pela Universidade Federal de Santa Maria (UFSM), é Mestranda do Programa de Pós-Graduação em Psicologia pela Universidade Federal de Santa Maria (UFSM) E-mail: cathelinerb@gmail.com

Jessica Vaz Lima, Especialista em Gestão e Atenção de Sistema Público de Saúde (Atenção Básica/ Estratégia Saúde da Família) pela Universidade Federal de Santa Maria (UFSM). Psicóloga Clínica em clínica privada no município de Caxias do Sul (RS).

E-mail: jessicavazlima@gmail.com

Pâmela Kurtz Cezar, Mestre em Psicologia pela Universidade Federal de Santa Maria (UFSM), é Psicóloga da Prefeitura Municipal de Santa

Maria (RS) - Secretaria de Município da Saúde (PMSM/SMS).

E-mail: pamelakurtz@gmail.com

Recebido em 11.Abr.16

Revisado em 13.Jan.17

Aceito em 19.Mai.17 